\title{
Integral field spectroscopy with optical fibres
}

\section{Vanderriest ${ }^{1}$}

\begin{abstract}
An efficient technique for obtaining complete spectral informations over moderately extended objects is to transform the geometry of their telescopic images to match a classical long "slit" aperture. This anamorphosis is conveniently made with bundles of optical fibres. Such devices are especially useful when high spatial resolution and large wavelength coverage are required at the same time. We review the existing instruments based on this technique and present some typical results. We discuss also the future developments of integral field spectroscopy with optical fibres (visible or IR domains) for the new generation of very large telescopes.
\end{abstract}

\section{Introduction:}

An obvious problem exists with the spectrographic observations of extended objects: we would like to gather informations on 3 variables $(\alpha, \delta$ and $\lambda)$ while the present detectors usually provide only 2 spatial dimensions (say, $x$ and $y$ ) for recording them. Except in the unrealistic case where the structure of the object exactly matches the shape of a spectrograph slit (it never happens...), we have to use time as the third dimension (scanning F.P., successive exposures with a long slit at different locations, etc...) or we have to pack 3 spatial dimensions into 2.

This geometrical transformation can be done either with an array of microlenses (in the TIGER approach) or with bundles of optical fibres (in the ARGUS approach). These are the 2 categories of Integral Field Spectrographs (IFS) presently in use. Whatever solution is chosen, the advantages are multiple:

- there is no need to worry about centering in a slit.

- there is no need to guess the seeing and choose accordingly a slit width at the time of observations.

(the position and size of the "entrance apertures" are defined a posteriori when processing the data, exactly as photometry can be done within any "aperture" on a bidimensional direct image with a CCD, for instance).

- there is no "slit effect" affecting the accuracy of radial velocity measurement; this property is inherent to the pupil mounting in TIGER and is verified with an excellent approximation in ARGUS due to light scrambling in the fibres.

${ }^{1}$ CFHT corp., USA; on leave from Observatoire de Pariz-Meudon (DAEC), FRANCE 
- spectra integrated over any area are obtained with a constant resolution, defined by the size of the fibres or lenslets (slicer effect).

- the spectrophotometric calibration from standard stars can be very accurate because there is no possibility of light losses (global or chromatic from differential refraction) due to centering inside a finite aperture.

\section{Some relevant properties of optical flbres:}

The idea of using bundles of optical fibres as a substitute for classical image slicers was suggested by Kapany in 1958.

In the past 10-15 years, multi-fibre spectrographs have been developped at a fast pace on many large telescopes for redshift surveys. But fibre optics instruments for area spectroscopy are still scarce. Besides technical conservatism, this could be due in part to practical difficulties for building efficient bundles.

In its path through an optical fibre, a light ray is subject to many total reflections at the boundary between the core and cladding glasses. The cladding thickness should be sufficient to avoid cross-talk between adjacent fibres or light leaking due to evanescent waves (fig. 1). At the same time, this thickness should be made as small as possible for maximizing the transmission, since light is poorly transmitted by the cladding. For the most current fibres having an index ratio $\simeq 1.1$ between core and cladding, a good compromise is a thickness around $5 \lambda$. For instance, a $100 \mu \mathrm{m}$ diameter fibre will have a $4 \mu \mathrm{m}$ cladding. Taking into account the inavoidable geometrical losses in packing such fibres together ( $9.7 \%$ for an hexagonal arrangement), the througput of a bundle could be $\sim 75 \%$.

Real fibres present also some focal ratio degradation (fig. 2a), i.e. beam broadening in excess of the diffraction limit set by the diameter of the fibre. The origin of this f.r.d. lies in diameter variations along the fibre and diffusion by imperfections at the core/cladding boundary. The effect is more or less important for fibres from different manufacturers. For the best ones, more than $90 \%$ of the light can be collected with an F/8 collimator from an F/8 entrance beam. Also, the effect is less for faster beams, so it would be more efficient to use fibres near their maximum acceptance. For an $\mathrm{F} / 3$ beam, for instance, f.r.d. is practically negligible.

For this reason, fibre optics integral field spectrographs recently designed for working with an $\sim F / 10$ collimator and at the Cassegrain or Nasmyth foci ( $\sim F / 10$ to F/15) of large telescopes will use additional input and output optics, so that the fibres "see" only an F/3 beam. At the same time, this could also allow to achieve a $100 \%$ coverage of the focal image (cf. FUEGOS design).

An interesting feature of the fibre optics solution for IFS is that the geometry at the output of the device is similar to a long slit (we call it a pseudo-slit), which means:

- Spectral range limited only by the size of the CCD

- Identical spectral coverage for each point in the field of view

- Good "legibility" of the raw data (see fig. 4-6) and rather classical reduction procedures

On the other hand, the maximum number of spectra that can be recorded with a given detector is smaller than in the TIGER solution. 


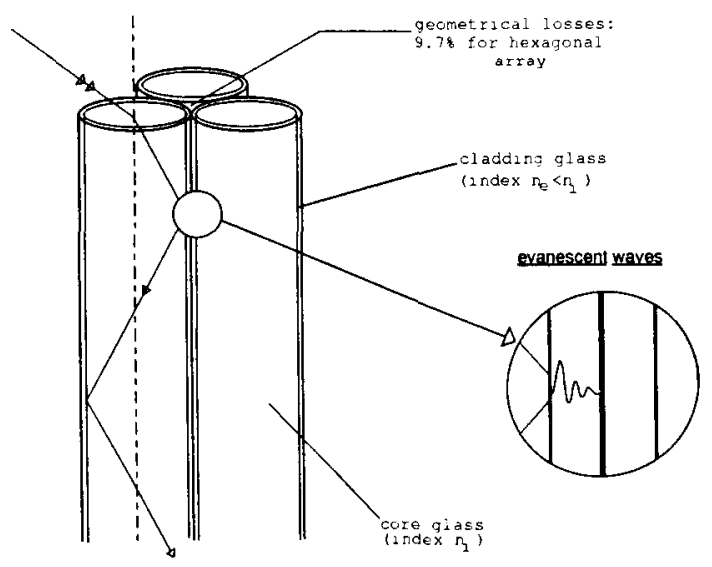

Figure 1. Role of the cladding in a fibre bundle

a)

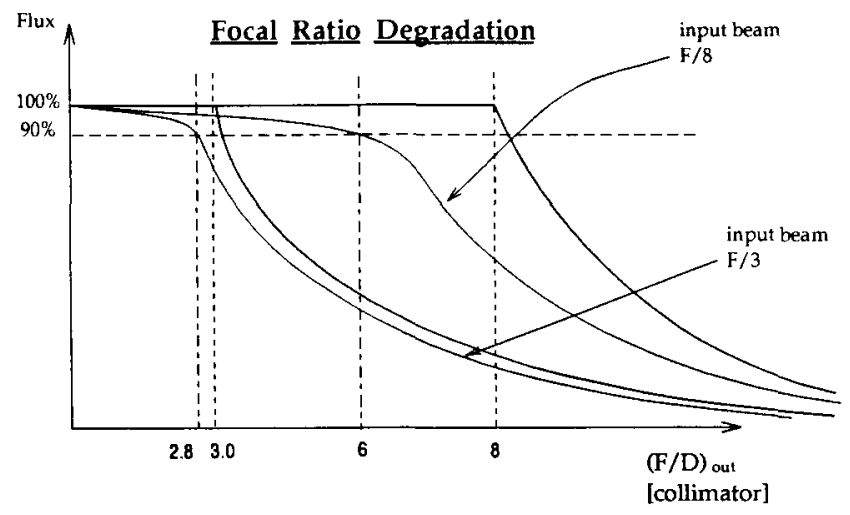

"Beam filling"

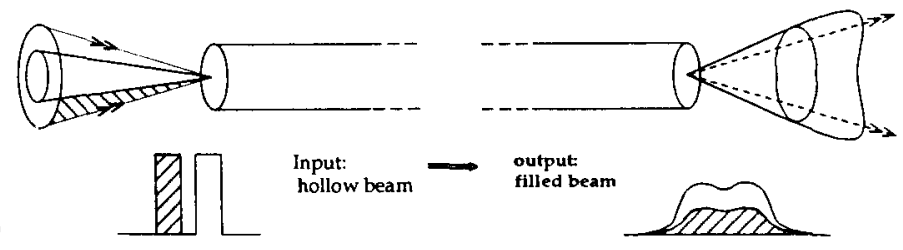

Figure 2. a) focal ratio degradation (f.r.d.) and b) "beam filling" effects in optical fibres 


\section{Existing instruments:}

Tests of optical fibres on the sky started at about the same epoch for multiobject spectroscopy (see e.g. Hill et al., 1980) and for integral field spectroscopy (Vanderriest, 1980) but the development of multi-object spectroscopy was faster. Similar applications for Infra-Red are just beginning now (Dallier et al., 1993, Levin et al., 1993). So, it is still possible to list the main IFS systems that have been in use so far, while it becomes hardly possible to do so for multispectroscopy systems.

- P. Gray did some observations on the sky with a prototype "fibre image slicer" (FIS) containing 100 fibres as early as 1981 with the AAT $3.9 \mathrm{~m}$ telescope (Gray et al., 1982).

- S. Barden used a small fibre bundle (49 fibres) dubbed DensePak with the KPNO $4 \mathrm{~m}$ telescope (Barden and Wade, 1988), but the system was not really used for science.

- Two fibre devices, Hexaflex (Arribas et al., 1991) and, recently, 2D-FIS (García et al., 1994) have been used with the $4.2 \mathrm{~m}$ William Herschel Telescope (WHT) and the $2.5 \mathrm{~m}$ Nordic Optical Telescope (NOT). The largest device has 125 fibres and scientific results obtained with these instruments have been published.

- Afanassiev and colleagues have used several IFS, either with micro-lens arrays or fibres on the $6 \mathrm{~m}$ telescope near Zelentchuk (Caucasus). They made the first attempt to combine a microlens array with a bundle of 225 fibres and used it successfully on the sky (Afanassiev et al., 1990; see also communications in these proceedings).

- The SILFID spectrograph (Vanderriest and Lemonnier, 1988), besides a multi-object spectroscopic mode and an imaging mode, used a 397-fibre fiber bundle for integral field spectroscopy (ARGUS mode). Various objects have been observed with it at the CFH telescope since 1986 .

- After the completion of the MOS-SIS double spectrograph and its commissioning at CFHT, P. Félenbok suggested that it would be easy to implement an IFS mode in it. The use of a flexible bundle (with 594 active fibres) allows to divert the light from the path it follows in other modes and to redirect it to the collimator after anamorphosis (fig. 3). The first observations in this mode (called MOS/ARGUS) took place in June 1993.

- Integral field spectroscopy in the IR was performed in January 1994 at CFHT with a bench spectrograph and a bundle of 62 fibres (J.-G. Cuby et al.).

The future developments of IFS on large telescopes will be shortly discussed as a conclusion of this review.

\section{Typical applications:}

The first tridimensional data obtained with a fibre optics spectrograph which were of real scientific use may well have been those gathered in December 1985 on comet Halley with SILFID and the $1.93 \mathrm{~m}$ telescope in Observatoire de Haute Provence (Malivoir et al., 1990). Since then, a large diversity of objects has been studied with IFS, from planets to quasars (see Vanderriest et al., 1994, for a list of targets observed at CFHT). 


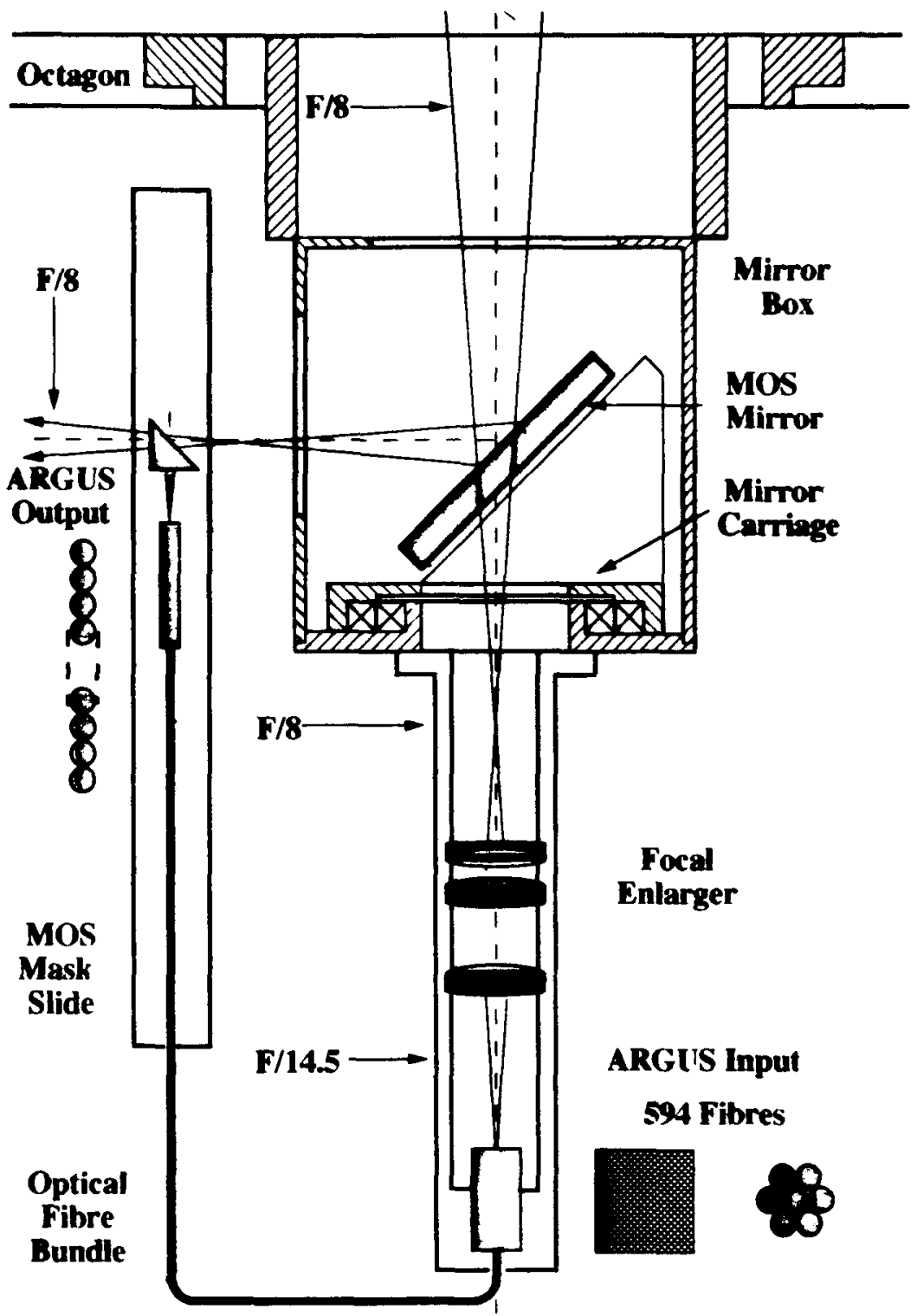

Figure 3. Sketch of the ARGUS device in the MOS spectrograph 


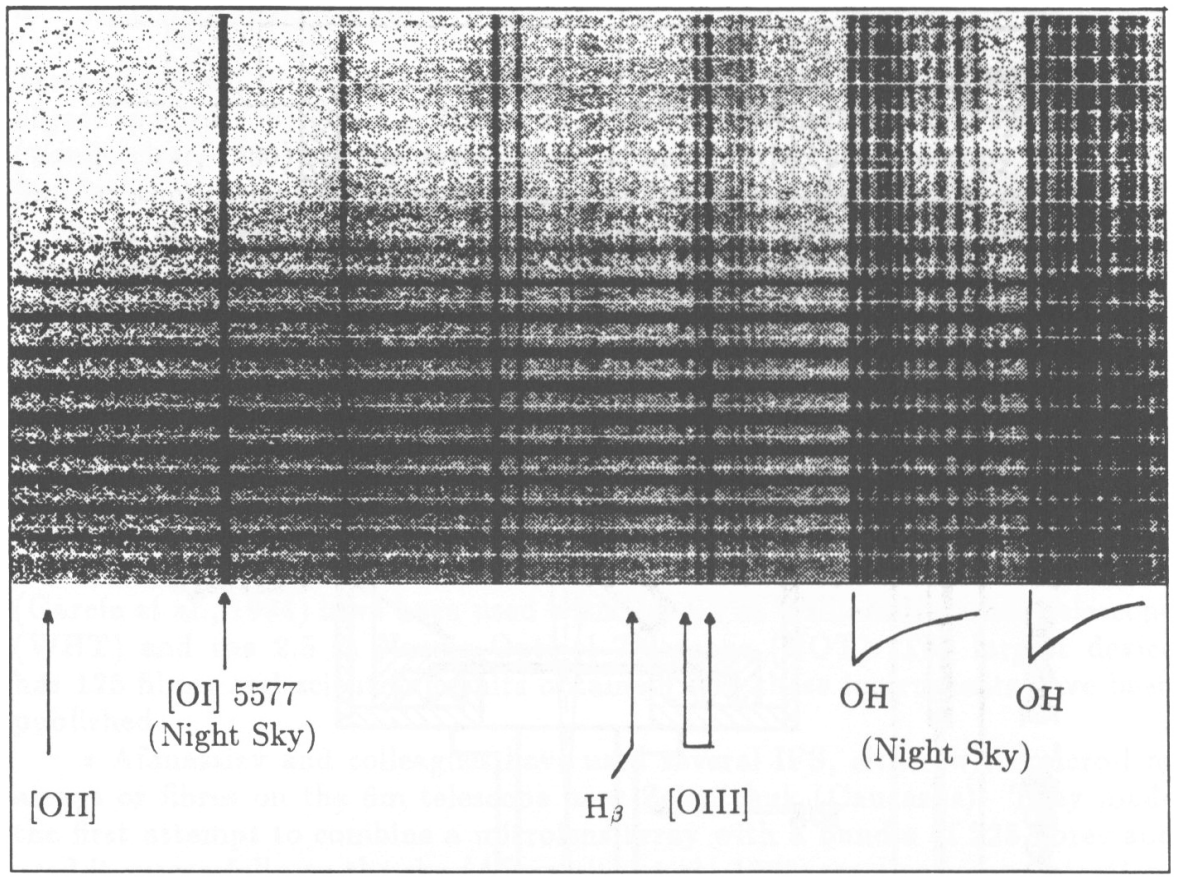

Figure 4. MOS-ARGUS observation of 4C 37.43: Fully calibrated bidimensional data frame.

As an example of application in the extragalactic domain, we present and discuss shortly the data obtained on the low-redshift quasar $4 \mathrm{C} 37.43(\mathrm{z}=$ 0.371) during the first run with the MOS/ARGUS device at CFH telescope in June 1993. Figure 4 shows the data calibrated in wavelength, spatial coordinates and flux but still in bidimensional format. Figure $6 \mathrm{~b}$ displays the reconstructed image in $[\mathrm{OIII}]_{5007}$ from extracted individual spectra. The main structures seen by Stockton and MacKenty (1987) on direct monochromatic images are recognizable, except that we had a poorer resolution (seeing was about 1.1"). The velocity field is complex, as can already be seen on the raw data. The East cloud is at $\sim-260 \mathrm{~km} . \mathrm{s}^{-1}$ with respect to the nucleus and presents a regular gradient (rotation?) while, in the $\mathrm{N}-\mathrm{W}$ cloud, the line is almost split into 2 components at some places, with the lowest velocity close to $-400 \mathrm{~km} \cdot \mathrm{s}^{-1}$. The variations of the $[\mathrm{OII}] /[\mathrm{OIII}]$ ratio observed across the extended emission line region correspond to a decreasing ionization with distance from the nucleus.

Two other quasars associated with clusters of galaxies, 3C281 ( $z=0.599)$ and $3 \mathrm{C334}(\mathrm{z}=0.555)$, have also been observed and present similar (although less spectacular) extended emission zones. In all the cases, the pressure deduced from the ionization ratio map suggests confinement by a hot intra-cluster medium (Crawford et al., in preparation). The total exposure time for each quasar was typically $5000 \mathrm{~s}$. 


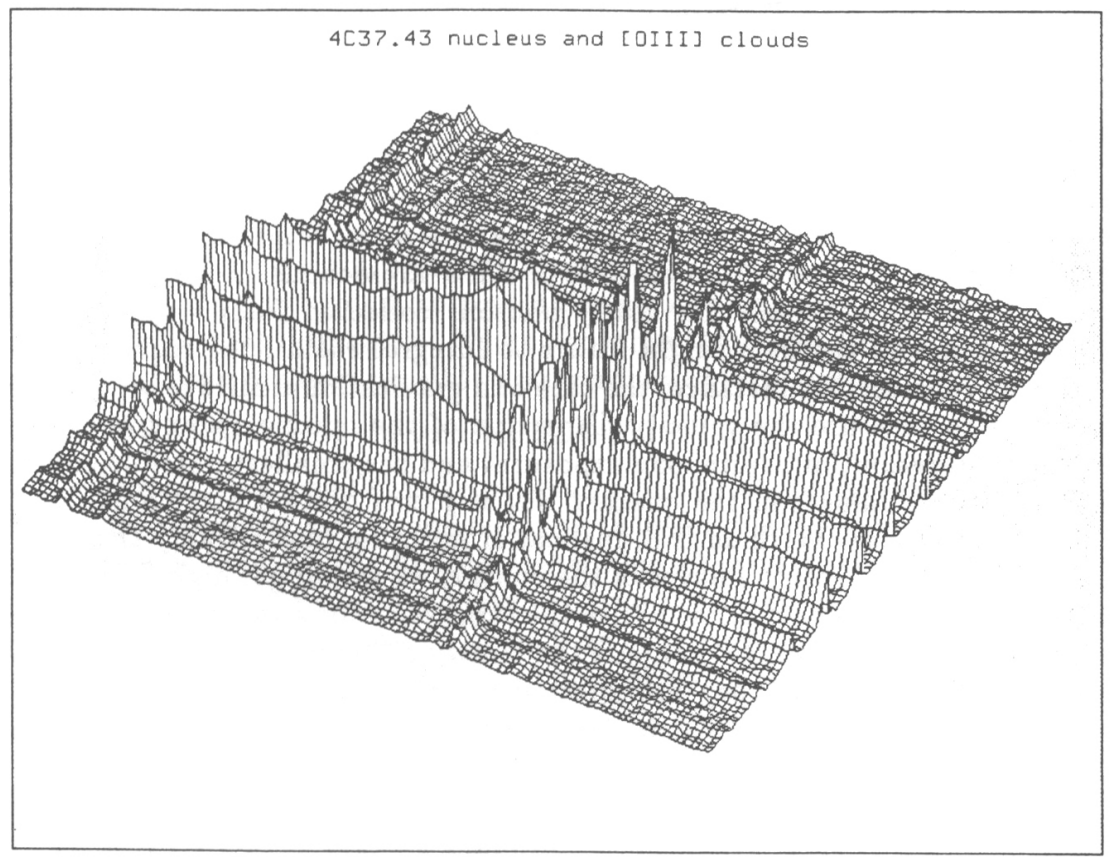

Figure 5. Perspective plot in the $\mathbf{H} \beta$ - [OIII] domain and around the nucleus. The narrow and broad components of nuclear $\mathrm{H} \beta$ are well visible, as well as the extent of extra-nuclear [OIII] emission.
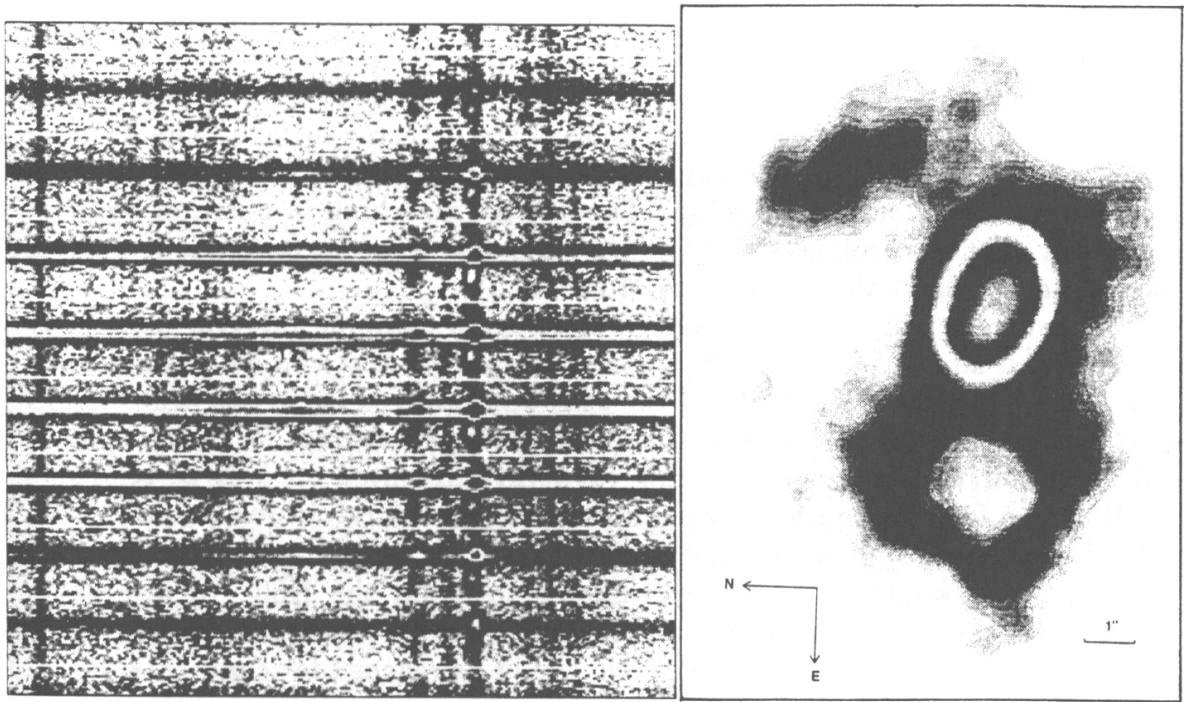

Figure 6. a) Isophotal plot in the center of the frame b) reconstructed image in $[\mathrm{OIII}]_{5007}$. 


\section{Conclusions and Perspectives:}

Still a young technique, integral field spectroscopy with optical fibres has produced interesting results in various astrophysical domains. The current trends in observational astronomy towards higher spatial resolution and fainter objects will probably hold for a while. So, it will be more and more usual to have to observe spectroscopically a faint object close to a much brighter one, or objects with complex structures within an area of a few arcseconds. Together with the concern for using optimally the costly photons collected from very large telescopes, this should ensure a bright future to Integral Field Spectrographs.

Let us consider, for instance, the case of a gravitational mirage on a quasar. If we want to measure the velocity dispersion of the lensing galaxy, we need a good spectral resolution, quite long exposures and the smallest possible contam. ination from the images of the quasar. But it seems quite a pity to use a long slit for that purpose. With an IFS, instead of rejecting unprofitably the light of the (comparatively) bright images of the quasar, we would get high $\mathrm{S} / \mathrm{N}$ spectra of all of them. This would allow to use the same data for another, totally different, purpose; for instance, to compare the emission line profiles for the different images and test the way they are affected by microlensing, which would give hints at the distribution and kinematics of BLR clouds in the quasar (Nemiroff, 1988).

With the development of large Infra-Red detectors and the very good transmission of fibres at such wavelengths, it would be easy to design IFS for this domain. In fact, an IR integral field mode will be implemented soon in the new (IR-improved) optics of the MOS-SIS spectrograph at CFHT. In 1996, this instrumental complex will offer possibilities for direct imaging, multi-slit spectroscopy and integral field spectroscopy at both visible and near-IR wavelengths, a unique facility as far as I know.

The association of microlens array (hexagonal pattern, for instance) with fibres is, in fact, the ideal solution for IFS: this allows at the same time a $100 \%$ coverage of the focal image and optimal use of the detector surface. The ARGUS mode of the FUEGOS spectrograph (Felenbok et al., 1994) which will be built for VLT-3 will use such a coupling. The precise matching in position between the pupils of the microlenses and the fibre tips has been mastered; it is made a little easier by the scale at the focus of the VLT.

Of course, it will be extremely interesting to combine integral field spectroscopy and adaptive optics. The number of spectra that can be distinguished goes like $s^{-2}$, where $s$ is the size of the spatially resolved elements. The OASIS spectrograph (see the review by R. Bacon in these proceedings for a description) will be the first instrument with this capability mounted on a large telescope (CFHT) in a favourable site. It will explore the spatial resolution domain down to 0.1 " with fields of view of a few arcsec.

Finally, it would be also very valuable to equip the HST -or any of its hypothetical successors- with IFS (Vanderriest, Courtès and Donas, 1984). With such devices, the 0.01 " resolution domain would be reached for compact objects.

Acknowledgments. I wish to thanks G. Courtès for his constant helps and encouragements during the heroic pre-SILFID times, as well as C. Crawford for permission to reproduce some data in advance of publication. 


\section{Discussion}

N. Douglas: You mentioned 2 properties of fibres, focal ratio degradation and " beam filling". A study in England recently published (in Gemini) shows that low f.r.d. and good beam filling are more or less mutually exclusive, so a trade-off is required there.

C. Vanderriest: Certainly, and this correlation is quite understandable. However, you can observe a good isotropization of skew light rays with quite a small degradation of the focal ratio. When choosing the fibres for making a device, we always favour the best possible preservation of focal ratio.

\section{J. Bland-Hawthorn: How do you flat-field your data ?}

C. Vanderriest: We usually make exposures on the dome lighted with a continuum lamp, using the same configuration than for the astronomical exposures. Flat-fielding is done on the bidimensional files after calibration in spectral and spatial dimensions. We approximate the true spectrum of the lamp by the average over all the lines of the CCD and we construct a normalized flat-field by dividing each line by this average. The method allows to correct all the local variations of the global sensitivity (pixel to pixel sensitivity + fibre to fibre transmission). It could lead to some inaccuracy in spectrophotometric calibration if large scale variations are important (systematic gradient of sensitivity, vignetting), which is not usually the case. Anyway, to alleviate this potential problem, we try to use the largest possible area of the detector for the exposures on spectrophotometric standards, by defocussing and/or drifting the image.

$K$. Jockers: What is the sampling on the CCD detector for the spectra of individual fibres? What about cross-talk introduced by light scattering within the spectrograph along the slit?

C. Vanderriest: With SILFID and a $15 \mu \mathrm{m}$-pixel CCD, the sampling was $\simeq 4$ pixels per resolved element (FWHM in either spatial or spectral direction); with MOS/ARGUS, it is reduced to $\sim 2.5$ pixels. Even with such a sampling, we do not have much trouble with light scattering. Remember that, due to the "image-slicer" geometry of the device, 2 adjacent pixels on the CCD along the spatial direction correspond to the same wavelength and also to adjacent points in the focal image, like for a classical long slit. So, if the object has no extreme gradient of brightness and is not undersampled by the fibres, there is no problem. With an undersampled image, we can make a simple correction to the spectra by subtracting a fraction of the flux from the adjacent fibre, calculated from the PSF of the spectrograph optics. Of course, the condition of spatial proximity is not verified when we jump from one row of fibre to the next one at the entrance pattern. To avoid excessive correction (as well as to make easier the calibration in spatial direction), we inserted in the exit "slit" dead fibres which separate the different rows. A more critical case is the Albireo spectrograph (see poster by G. Herpe), for which the sampling on the CCD is only 2 pixels per fibre. So, we inserted one spacer between each fibre in the exit "slit" for the ARGUS mode of this instrument.

B. Rocca-Volmerange: What magnitude limit could you hope for extended objects when emission lines are absent or subtracted from the spectrum? 
C. Vanderriest: I cannot give a very precise value, because it depends so much on the surface brightness of the object, the strength of its absorption lines and the spatial sampling with the fibres. But, as an example, we measured the redshift $(z \simeq 0.4)$ of a galaxy with $V=21$ in 2 hours, with a spatial sampling of $0.7^{\prime \prime} /$ fibre (see Haddad and Vanderriest, 1991). The galaxy was spread on about 8-10 fibres. It was observed with SILFID and a photon counting camera of quite low efficiency in 1987. Now, with MOS/ARGUS, you can expect to measure a similar object at magnitude 22 in a reasonable time with a thick CCD and near magnitude 22.5 with a thinned CCD.

\section{References}

Afanasiev V., Vlasiuk V., Dodonov S., Silchenko O. (1990) Academ. Nauk. USSR, preprint 54 .

Arribas S., Mediavilla E., Rasilla J.-L. (1991), ApJ, 369, 260.

Barden S., Wade R. (1988) ASP Conf. Ser. 3, 113.

Dallier R., Baudrand J., Cuby J.-G. (1993) ASP Conf. Ser. 37, 310.

Félenbok P., Cuby J.-G., Lemonnier J.-P., Baudrand J., Casse M., André M., Czarny J., Daban J.M., Marteaud M., Vola P. (1994) SPIE Symp. "Astron. Telescopes \& Instrum. for the $21^{\text {th }}$ century", $2198,115$.

García A., Rasilla J.-L., Arribas S., Mediavilla E. (1994) SPIE Symp. "Astron. Telescopes $\mathcal{G}$ Instrum. for the $21^{\text {th }}$ century", 2198, 15.

Gray P., Phillips M., Turtle A., Ellis R. (1982) Proc, Astr. Soc. Austr., 4, 477. Haddad B., Vanderriest C. (1991) A\&A, 245, 423.

Hill J., Angel J., Scott J., Lindsley D., Hintzen P. (1980) ApJ, 242 L.69.

Kapany N. (1958) in "Concepts of classical Optics", J. Strong ed., Freeman publish. (San Francisco).

Levin K., Tran D., Kindler E., Glenard D., Joyce R. (1993) ASP Conf. Ser. 37, 295.

Malivoir C., Encrenaz T., Vanderriest C., Lemonnier J.-P., Kohl-Moreira J.-L. (1990) Icarus 87, 412.

Nemiroff R. (1988) ApJ, 335, 593.

Stockton A., MacKenty J. (1987) ApJ, 316, 584.

Vanderriest C. (1980) PASP, 92, 858.

Vanderriest C., Bacon R., Georgelin Y., LeCoarer E., Monnet G. (1994) SPIE Symp. "Astron. Telescopes \& Instrum. for the $21^{\text {th }}$ century", 2198, 1376.

Vanderriest C., Courtès G., Donas J. (1984) J. Optics (Paris), 15, 237.

Vanderriest C., Lemonnier J.-P. (1988) in "Instrumentation for ground-based Astronomy" (IX ${ }^{\text {th }}$ Santa-Cruz workshop), ed. L. Robinson, p. 304, Springer-Verlag. 